\title{
On-Line Educational Tool for the Parallel Operation of the Synchronous Generators
}

\author{
ERDAL BEKIROGLU, ${ }^{1}$ ALPER BAYRAK ${ }^{2}$ \\ ${ }^{1}$ Faculty of Engineering and Architecture, Abant Izzet Baysal University, Bolu, Turkey \\ ${ }^{2}$ Faculty of Engineering, Izmir Institute of Technology, Izmir, Turkey
}

Received 12 January 2009; accepted 18 March 2009

\begin{abstract}
This study presents a PC and a PIC microcontroller based an on-line educational tool for the monitoring and controlling of the parallel operation of the synchronous generators. The presented tool measures the voltages, the frequencies, the phase sequences, and the synchronism time data of the generators through microcontroller. The measured data, the voltage graphics, and the status of the parallel operation conditions are monitored on the computer instantaneously. The parallel operation of the generators is controlled with the developed tool automatically. The educational tool is user-friendly, real-time, reliable, and precise. (c) 2009 Wiley Periodicals, Inc. Comput Appl Eng Educ 19: 669-677, 2011; View this article online at wileyonlinelibrary.com/journal/ cae; DOI 10.1002/cae.20351
\end{abstract}

Keywords: educational tool; visual interface; synchronous generators; parallel operation; microcontroller

\section{INTRODUCTION}

Electrical power systems consist of the interconnection of several synchronous generators (SGs) operating in parallel, interconnected by transmission lines, and supplying large numbers of widely distributed loads. When an SG is connected to an interconnected system containing many other SGs, the voltage and the frequency at its terminals are fixed by the system [1]. Operating multiple generators in parallel has some important advantages such as; increased reliability, expandability, flexibility, serviceability, and efficiency. The redundancy inherent in parallel power generation provides significantly greater reliability for critical loads. In a parallel configuration, if one generator fails, the most critical loads are redistributed among the other units in the system. Utilizing multiple smaller generators instead of a single large generator offers greater location flexibility. With multiple generators available, individual units can be taken out of service for repair or maintenance without losing standby power for critical circuits. The parallel operation allows the SGs operating around their rated load resulting operating with high efficiency [2].

Variety studies related to the parallel operation and the control of the SGs have been presented in the literature. These studies have been focused on the simulation, the power control,

Correspondence to E. Bekiroglu (bekiroglu_e@ibu.edu.tr). (C) 2009 Wiley Periodicals Inc. the synchronization, and the stability of the SGs [3-4]. A standard of the synchronization used for the power systems has been given in Ref. [5]. The simulation of the parallel connected SGs has been presented in Ref. [6]. An automatic digital synchronization system using sensors and the PLC has been proposed in Ref. [7]. A digital control and monitoring system for a turbine and an SG has been presented in Ref. [8]. Four sensors based on microcomputers are used for the measurements. An experiment test set-up of the SGs for the educational purposes is designed and implemented in Ref. [9]. The study is focused on the hardware and the microcontroller parts of the system. A microprocessor-based automation laboratory for power system has been described in Ref. [10]. The principles of microprocessor-based excitation regulator and synchronization control device have been presented in the study.

Educational tools have important role for engineering education to improve the efficiency of the training. Several educational tools have been presented for the electrical machinery courses and related subjects in the recent years. Computer-based virtual educational tools have been proposed for the electrical machinery course and laboratory [11-13]. An integrated virtual learning system for the development of the motor drive systems has been developed [14]. A virtual instrument to measure and detect the spatial saliencies in induction motors has been presented [15]. The DC motor speed control methods and induction motor tests using MATLAB/Simulink have been presented for the undergraduate electrical machinery courses $[16,17]$. Web-based remote on-line maximum wind power 
monitoring and control system has been proposed in Ref. [18]. The virtual instrument tool presented in the study can carry out an on-line maximum wind power tracking using the LabVIEW and the microprocessor. A virtual electrical drive control laboratory consists of neuro-fuzzy control of induction motors has been presented in Ref. [19]. A software tool that offers students a quick and easy introduction to simulation and lab experiments related to electrical machines and power electronics has been described in Ref. [20]. An educational tool for neural network control of brushless DC motors has been presented in Ref. [21]. Computerbased educational tool of the PWM used for the static converters is presented in Ref. [22]. Computer-based educational tools have been proposed for the fault analyses and synchronization of the power systems. An interactive laboratory experiment that provides students to examine the effect of fault conditions on a power system in a realistic manner has been designed in Ref. [23]. Virtual instrument-based synchronization system has been presented in Ref. [24]. Data acquisition cards have been used in these two studies to process the signals of the systems.

In this study, a PC and a PIC16F877A microcontroller based an on-line educational tool has been proposed for the parallel operation of the SGs. The proposed tool measures all data of the hardware system through the microcontroller on-line and sends the measured data to the PC for the visualization and the control processes. The microcontroller part of the system reads, calculates, and evaluates the frequencies, the voltages, and the phase sequences of the SGs and then provides the synchronization for the parallel operation of the generators. The program coded into the microcontroller has been developed effectively to simplify the interface electronics circuit of the system. The visual interface has been developed for monitoring the measured data, the graphics, and the instant status of the parallel operation conditions. The parallel operation of the generators is achieved automatically with the control button of the educational tool when all parallel connection conditions occur. There is no need to use measuring devices, such as voltmeters, frequency meters, phase sequence meter, and synchronoscope for measuring and observing the parallel connection conditions. The proposed educational tool is on-line, reliable, stable, and accurate to be used for the measuring, monitoring, and parallel operating of the SGs. The proposed tool is very convenient for training of the parallel operation of the SGs for the undergraduate students, engineers, and technicians. The educational tool with experimental test set-up will precisely help users to understand the parallel operation of the SGs. It can be easily and effectively used for both the industrial and the educational purposes.

\section{PARALLEL OPERATION OF THE SGs}

The SGs are connected in parallel to feed bigger loads and improve reliability of the electrical power systems. When the SG is connected to a large interconnected system containing many other SGs, the voltage and the frequency at its terminals are substantially fixed by the system. The fields of the stator and the rotor must rotate at the same speed, and therefore the rotor must rotate at precisely synchronous speed for the production of a steady unidirectional electromagnetic torque [1]. The SG can be connected in parallel with another SG or with transmission line when required conditions occur. The conditions required for the parallel connection of the SGs are given as follows:
(1) Frequencies at their terminals should be equal $\left(f_{1}=f_{2}\right)$.

(2) Voltages at their terminals should be equal $\left(V_{1}=V_{2}\right)$.

(3) Phase sequence of the SGs should be the same. In the other words, the direction of the rotating field should be the same.

(4) Parallel connection should be realized at the synchronism time.

\section{Frequency Equality}

Parallel operated SGs should rotate exactly at the same speed for the same pole numbers to produce equal frequencies. If the frequencies of the SGs are not equal, the speed of their rotating machines should be adjusted. The equation of the frequency is given as follows:

$$
f=\frac{n P}{120}
$$

where $f$ is the frequency of the terminal voltage of the SG in $\mathrm{Hz} ; n$ the rotational speed of the rotating machine of the SG in rpm; $P$ the pole numbers of the SG.

\section{Voltage Equality}

The SGs should have equal terminal voltages for the parallel operation. The terminal voltage generated from the SG is given as follows:

$$
E_{\mathrm{rms}}=\sqrt{2} \pi f k w N_{\mathrm{ph}} \phi
$$

where $E_{\mathrm{rms}}$ is the terminal voltage of the SG per phase; $k w$ the winding coefficient; $N_{\mathrm{ph}}$ the serial turn number per phase; $\Phi$ the flux er pole.

As seen from the equation, the flux should be adjusted to generate demanded terminal voltage. The flux can be adjusted easily by adjusting the excitation current of the field winding of the SG.

\section{Phase Sequence Equality}

Phase sequence of the SGs, in the other words, produced rotational magnetic fields of the SGs should be in the same direction. If the phase sequences of the SGs are not the same, the location of the two phase of any SG or the rotating direction of any SG should be changed to provide equality of the phase sequence.

\section{Synchronization}

If the parallel connection conditions described above are provided, the SGs can be paralleled on the synchronism time. The synchronism time means that there is no phase angle between the phase voltages of the two SGs.

In the classical parallel operation systems; the frequencies are measured with the frequency meters, the voltages are measured with the voltmeters, the phase sequence is measured with the phase sequence meter, and the synchronism time is measured with the synchronoscope. The block diagram of the parallel operation system with classical measurement devices is given in Figure 1. 


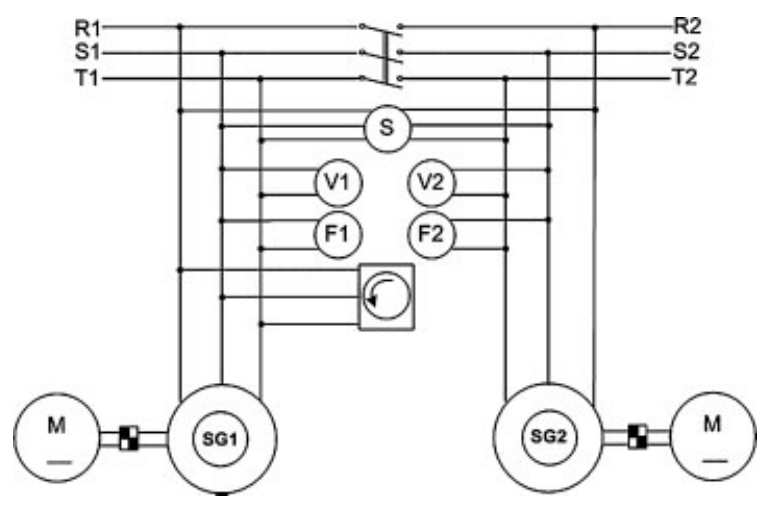

Figure 1 Block diagram of the classical parallel operation of the two SGs.

\section{ON-LINE EDUCATIONAL TOOL FOR THE PARALLEL OPERATION OF THE SGs}

A PC and a PIC microcontroller based an educational tool has been developed for monitoring and controlling of the parallel connection of the SGs. The block diagram of the parallel connection system with the proposed control tool is shown in Figure 2. Signal transformers are used as voltage sensors. The microcontroller receives voltage signals of the SGs and calculates the voltages, the frequencies, and the phase sequences. It determines the parallel connection time when all conditions are provided. The microcontroller sends the data of the system to the PC. The parallel operation is controlled from the educational tool on the PC. The PC sends the command to the microcontroller and then the microcontroller produces signal for the relay realizing the parallel operation of the SGs. In the presented study, the measurement devices used in the classical parallel connection system are eliminated. Compact, quick, and precise a microcontroller-based measurement card has been designed. The microcontroller card measures all the required data exactly and controls the parallel operation of the SGs. The overall view of the designed and tested system is given in Figure 3. The system is mainly composed from the hardware system including two SGs unit, the microcontroller card, and the visual interface for the educational tool.

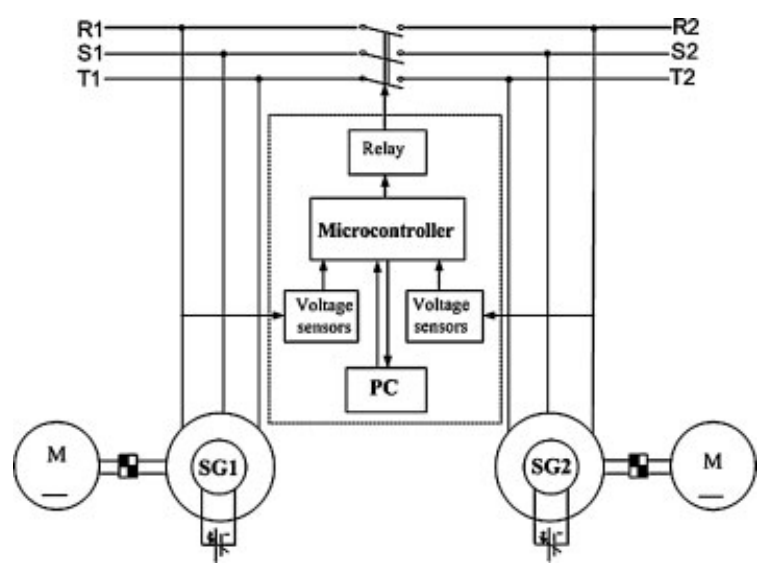

Figure 2 Block diagram of the educational tool controlled system.

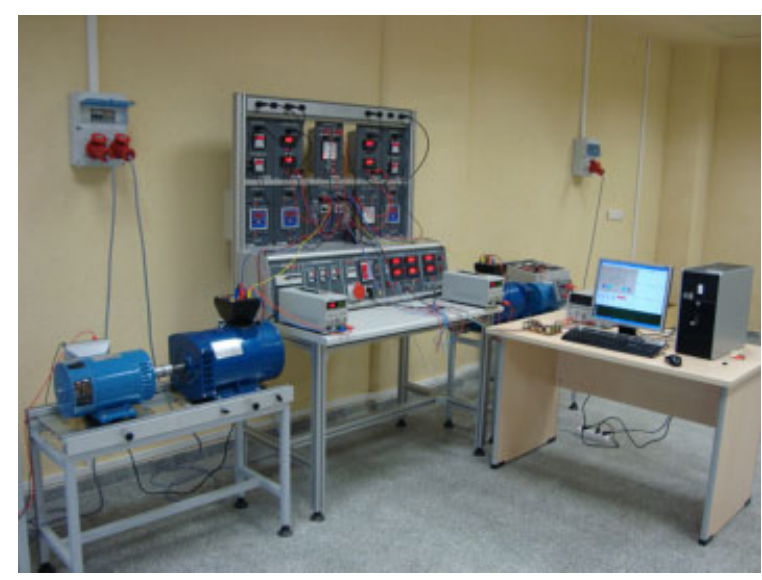

Figure 3 View of the overall parallel operation system.

\section{Hardware}

Two SGs (4-pole, $4 \mathrm{~kW}$ ) rotated with the DC motors are used for the experimental test set-up of the parallel operation system. The frequencies of the voltages at terminals of the SGs are adjusted by the speed control of the DC motors. The frequency of the phase voltages of the SGs are obtained as $50.0 \mathrm{~Hz}$ when the DC motor is rotated with the $1,500 \mathrm{rpm}$. The amplitude of the SGs voltages are adjusted by the power supply connected to field winding of the SGs. A 3-phase parallel connection switch is used to implement the parallel connection of the SGs.

\section{Microcontroller Card}

A single 16F877A PIC microcontroller has been used in the control system. The microcontroller has been operated with $4 \mathrm{MHz}$ oscillator. The loop time of the each command is $1 \mu \mathrm{s}$. The comparator, the comparator voltage reference, the ADC, the Timer-1 modules, and the A, B, E ports of the microcontroller have been used in the application. The port configuration of the microcontroller is given in Figure 4. The ports $\mathrm{A}$ and $\mathrm{E}$ have been set as analogous inputs and the port-B is set as digital output. R1 and R2 phase signals of the SGs are connected to the number 0 and 1 inputs of the port-A, T1 and T2 phase signals are connected to the number 3 and 5 inputs of the port-A, S1 and S2 phases are connected to the number 0 and 1 inputs of the port-E, respectively.

The actual phase voltages of the SGs are converted with the $220 / 5 \mathrm{~V}$ signal transformers to be processed with the microcontroller. The microcontroller reads, calculates, and evaluates the frequency, the voltage, and the phase sequence of the received input signals and then provides the synchronization for the parallel operation of the generators. The PIC is connected to the PC through RS232 communication port in 9,600 baud. When the PIC sends a data, the PC reads and processes the data and then sends command to the PIC for transmitting the next data. PIC waits for the command and sends the next data. If all the conditions are satisfied for parallel connection, the PC sends parallel connection command. When the command is received by the microcontroller, it waits for the synchronization moment and when it caught the synchronization moment, it close the relay connected to the fourth pin of the port-B.

The comparator, the comparator voltage reference, and the Timer1 modules of the microcontroller are used for the 


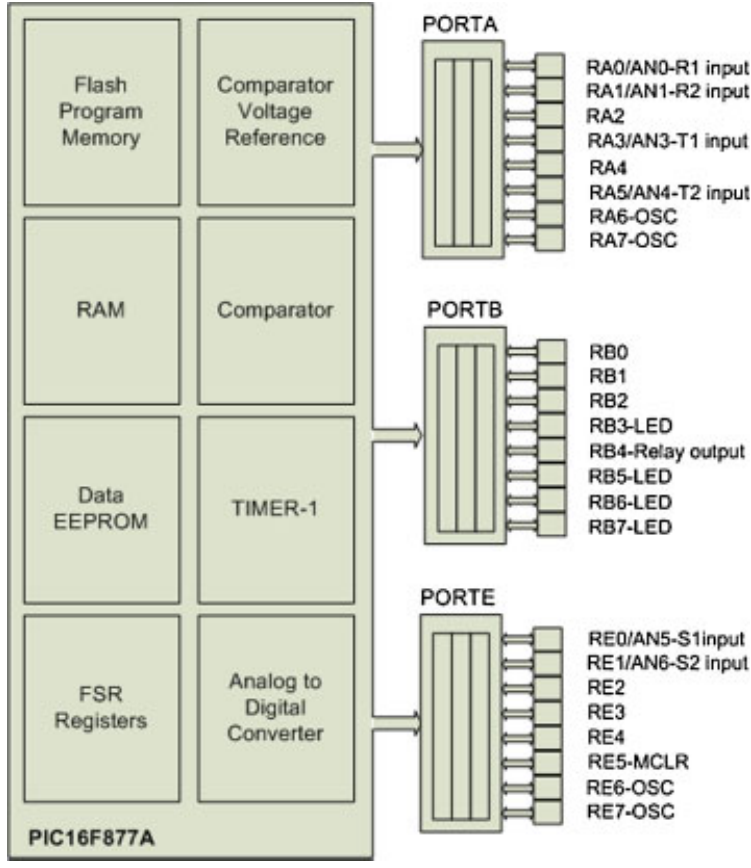

Figure 4 Port configuration of the microcontroller.

measurement of frequency. Sinusoidal phase voltage signals of the SGs are compared with internal produced reference voltage $V_{\text {ref }}=0$ and then converted to the square wave signals. Period of the signals are identified from this square wave signals by using Timer1, and then frequency of the signals are calculated. The ADC module of the microcontroller is used for the voltage measurements. The microcontroller receives approximately 300 samples during a period of the voltage signal. Each received sample compared with the previous sample and the biggest value is saved as a peak value of the half period of the input signal. Then the rms value of the voltage is calculated. The ADC module of the microcontroller is also used for the voltage measurements determination of the phase sequence. The signals received from the $\mathrm{R}$ and the $\mathrm{S}$ phases of the generators are used for the determination of the phase-sequence. The received signals are converted to the square wave signals. According to the calculation, the controller determining the direction of the phase sequence as 0 or 1 . The comparator, the comparator voltage reference, and the Timer1 modules of the microcontroller are used to capture synchronism time between the phase voltages of the two SGs. The signals received from the $\mathrm{R}$ phases of the generators are used for the detection of the synchronization. Both the $\mathrm{R}$ phases of the generators are converted to the square wave signals. The synchronism time is captured when the two phases are rising from 0 to 1 at the same time. The frequency, the voltage, and the phase sequence of each SG are measured, respectively, and when all the conditions occur, the synchronism time is searched to achieve parallel operation of the SGs. Each parallel connection condition has been determined by the sub-routine of the software program coded into the microcontroller.

A photograph of the designed microcontroller card connected to the RS-232 serial communication port of the $\mathrm{PC}$ is given in Figure 5. Three transformers are used as voltage sensors for each SG. The LEDs are placed to observe communication and the

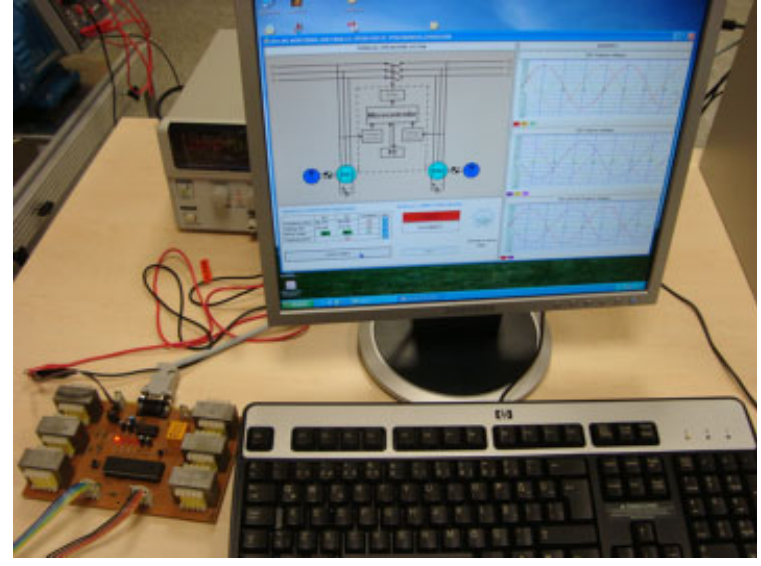

Figure 5 View of the microcontroller card connected to the PC.

data transfer. The relay is used for the implementation of parallel operation of the SGs. Microcontroller card provides all required measurements and control processes according to the commands incoming from the PC.

\section{Visual Interface for the Educational Tool}

The educational tool is designed for monitoring the data obtained from the hardware and controlling the parallel operation of the SGs. The visual interface of the educational tool developed with the C\# software tools of Visual Studio .NET platform. The designed visual interface is illustrative and user-friendly. The visual interface software communicates with the microcontroller on-line. The flowchart of the software program developed for the educational tool is given in Figure 6. While the SGs are operated and the microcontroller and the PC are turned-on, the developed tool is ready to receive data from the system. When the port of the PC is opened, the data of the system are transferred to the PC and showed on the educational tool. The received data are calculated, compared, and then saved. The data collected from the SGs are compared for each parallel connection conditions. The data are also transmitted to the graphics window. When all conditions are provided, the educational tool can send parallel operation command or continue to receive data from the system. The monitored and processed data are on-line data of the hardware system. The developed tool achieves all measurement and control processes as real-time.

The general view of visual interface part of the developed educational tool is given in Figure 7. This interface will be called as educational tool in the following sections. The educational tool mainly consists of four parts, which are: (a) Parallel operation system, (b) Parallel operation conditions window, (c) Parallel connection control panel, and (d) Graphics window. The parts of the educational tool are described as follows:

Parallel Operation System. The main block diagram of the parallel operated SGs with the control block diagram is given in this part. When the parallel operation is not implemented, the parallel connection switch is open. This switch will be closed when the SGs are operated in parallel.

Parallel Operation Conditions Window. The parallel operation conditions are monitored on this part. The on-line frequencies, the 


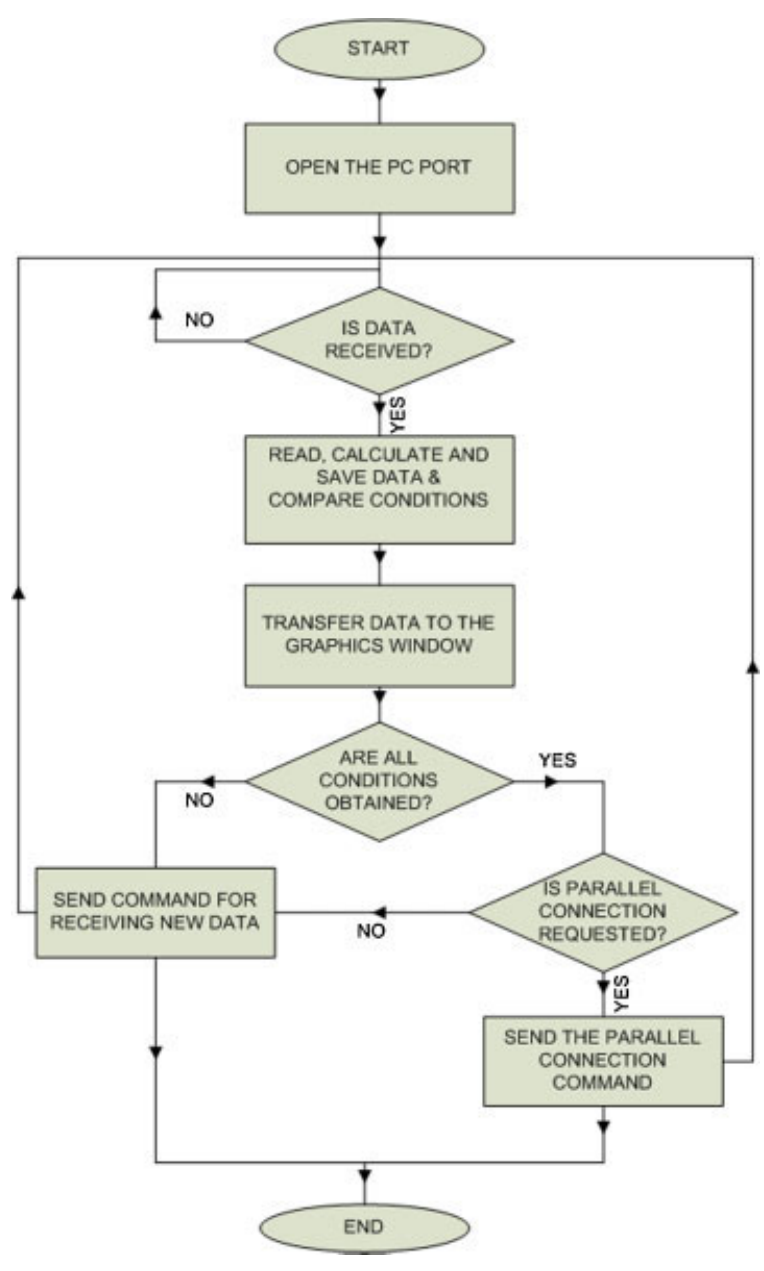

Figure 6 The flowchart of the educational tool.

voltages, the phase sequences, and the synchronism data of the hardware system are showed on this part. The Correct $\ominus$ and the Stop $\approx$ signs are used for the monitoring the actual status of each condition. When the conditions are provided the Correct sign, otherwise the Stop sign is showed. Users can access the information about each condition by clicking the Info is button. When this button is clicked, related information window is opened. This option helps the users to improve their information related to the parallel connection conditions.

Parallel Connection Control Panel. The parallel operation control of the system is implemented in this part. The Connect and the Disconnect buttons are located on this part. If the parallel connection conditions are not obtained, the Connect button is not active and its color is red. When all the parallel operation conditions are obtained, the Connect button becomes active and its color will be green. In this case, when the Connect button is clicked the SGs are connected in parallel and also the parallel connection switch on the educational tool is closed at the same time. The Disconnect button is used to cancel the parallel operation of the SGs.

Graphics Window. On-line voltage graphics of the SGs are showed in this part. The graphics window is divided into three divisions. First division shows the 3-phase voltage graphics of the SG1, the second division shows the 3-phase voltage graphics of the SG2. The R phases of the SG1 and the SG2 are showed on the third division to show the phase angle (synchronization) between the voltages.

Also the Open Port and the Help buttons are placed on the educational tool. The Open Port button is clicked to start receiving the data from the hardware system. The Help button is used for the users to access the theoretical information about the parallel operation of the SGs and helps how to use the educational tool.

\section{EXPERIMENTAL RESULTS}

The experimental test system has been operated for the different working cases to present the ability of the educational tool. Some of the experiments are taken from the system have been given in this part.

\section{Case 1}

The SGs are rotated with the DC motors with the defined rotating speed. The voltages, the frequencies, and the phase sequences data of the SGs are measured with the microcontroller and monitored on the educational tool. We clicked to the Open Port button to receive and show the data of the system. The view of the educational tool with real data of the hardware system is shown in Figure 8. The frequency of the SG1 is $47.501 \mathrm{~Hz}$ while the frequency of the SG2 is $50.100 \mathrm{~Hz}$. The phase voltage of the SG1 is $213.33 \mathrm{~V}$ while the phase voltage of the SG2 is $220.86 \mathrm{~V}$. The frequency and the voltage equivalent conditions are not provided in this case. Thus, the Stop sign appears for the frequency and the voltage conditions as seen from the figure. The phase sequences of the SGs are in the same direction and the Correct sign is appeared for the phase sequence condition as seen in the Phase Order raw. Since the voltage and the frequency equivalent conditions are not provided, we cannot search for the synchronization time. Consequently, we cannot connect the SGs in parallel. The Connect button is not active and it is Red in this case. The 3-phase voltages of the SG1 and the SG2 are plotted on the graphics part of the tool. Also, one phase of the each SG is plotted to see phase angle and to observe the synchronization between the two SGs.

\section{Case 2}

The phase sequence of the SG2 is changed to test the educational tool for the phase sequence condition. The obtained data in this case are monitored on the educational tool and presented in Figure 9. The frequency of the SG1 is $50.050 \mathrm{~Hz}$, while the frequency of the SG2 is $50.100 \mathrm{~Hz}$. The voltage of the SG1 is $220.43 \mathrm{~V}$ and the voltage of the SG2 is $220.86 \mathrm{~V}$. The frequency and the voltage equality conditions are provided. However, the phase sequences of the SGs are not equal and the direction is showed in the opposite directions on the educational tool as seen from this figure. The Stop sign is appeared on the Phase Order raw in this case. Consequently, we cannot connect the SGs in parallel. The Connect button is still not active and it is Red as in the Case 1. The Info sign is clicked, the Phase Sequence Equality information window is opened to help the user for how to provide this equality. 


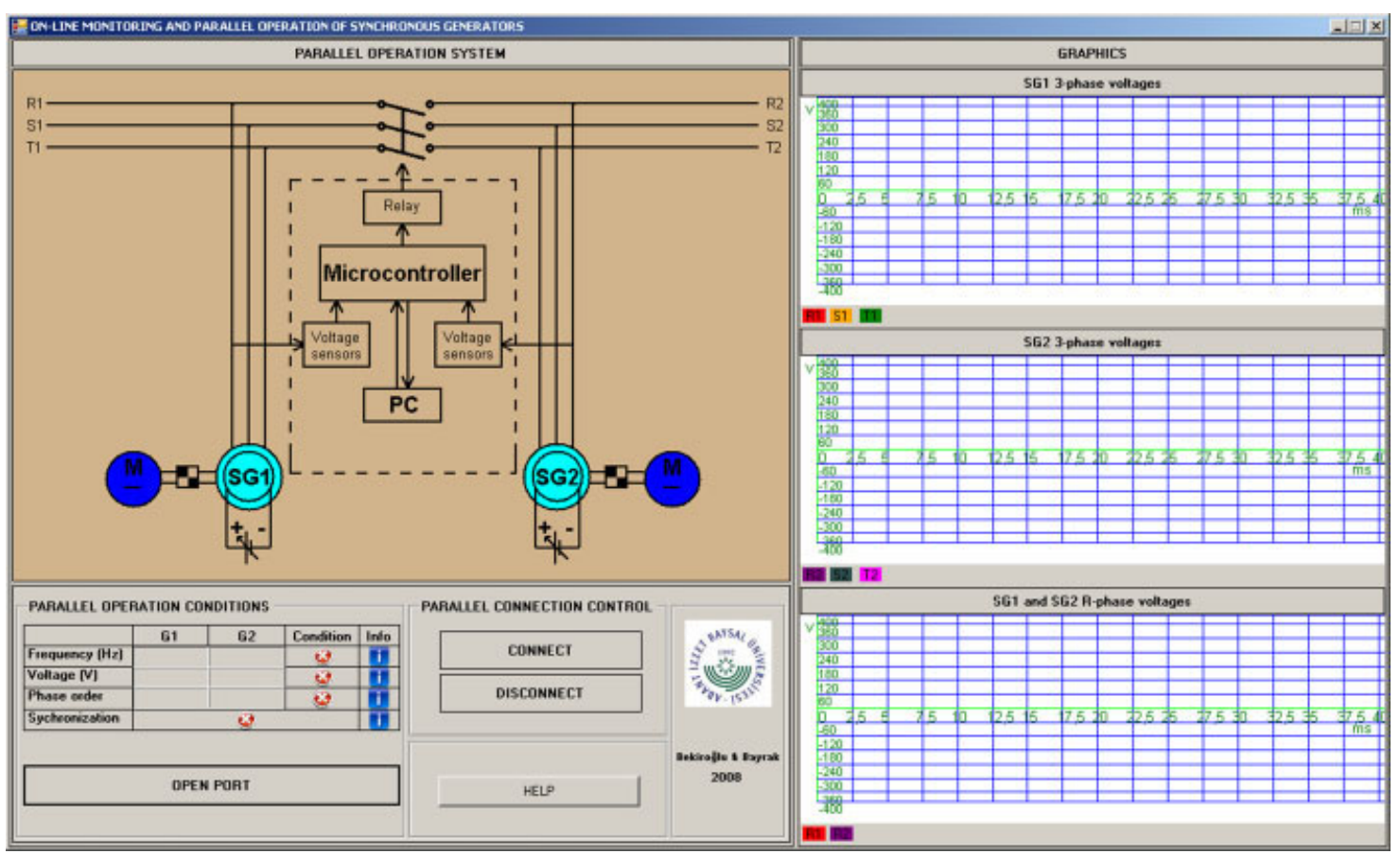

Figure 7 General view of the educational tool. [Color figure can be viewed in the online issue, which is available at wileyonlinelibrary.com.]

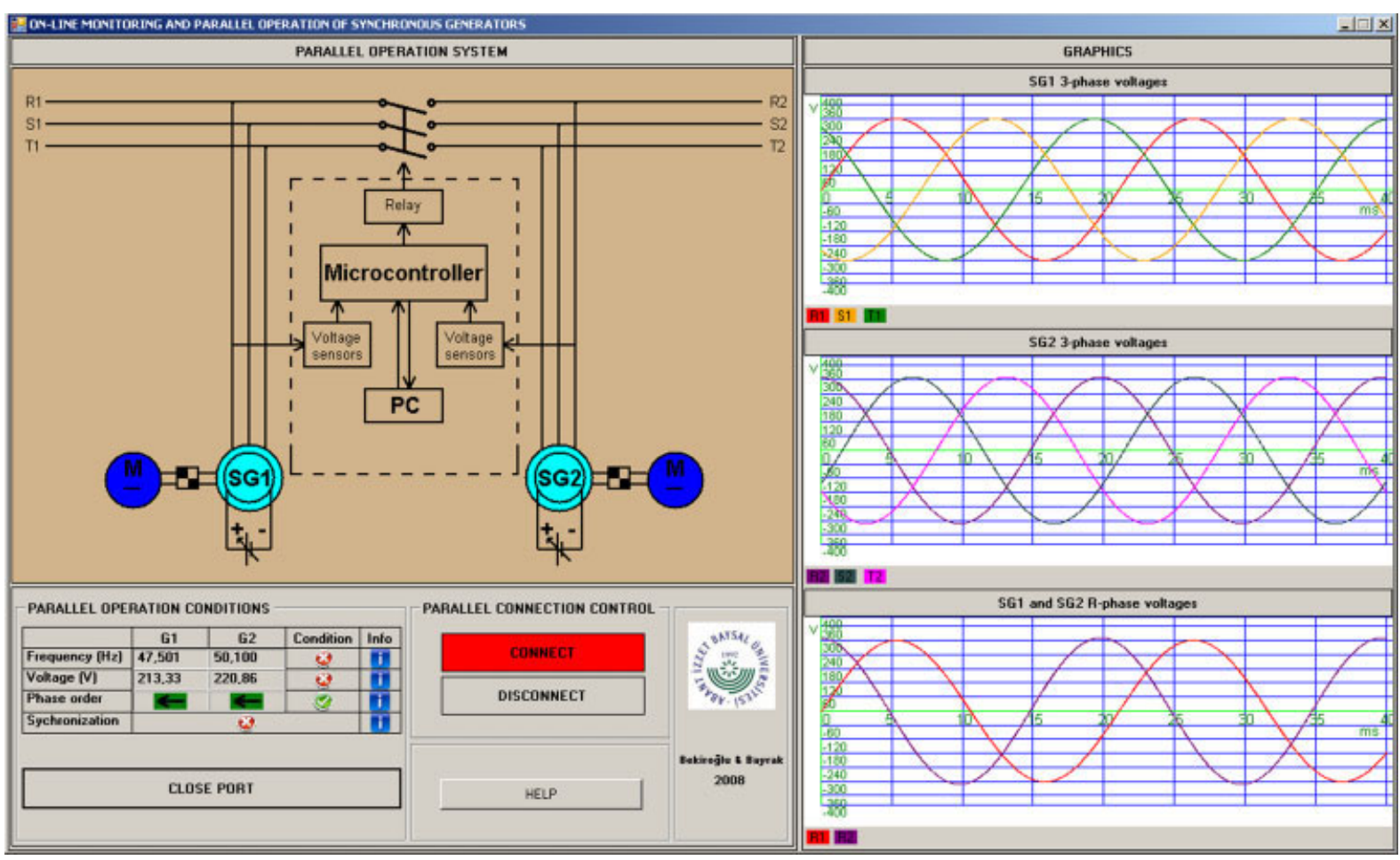

Figure 8 View of the educational tool with on-line data of the hardware system (Case 1). [Color figure can be viewed in the online issue, which is available at wileyonlinelibrary.com.] 


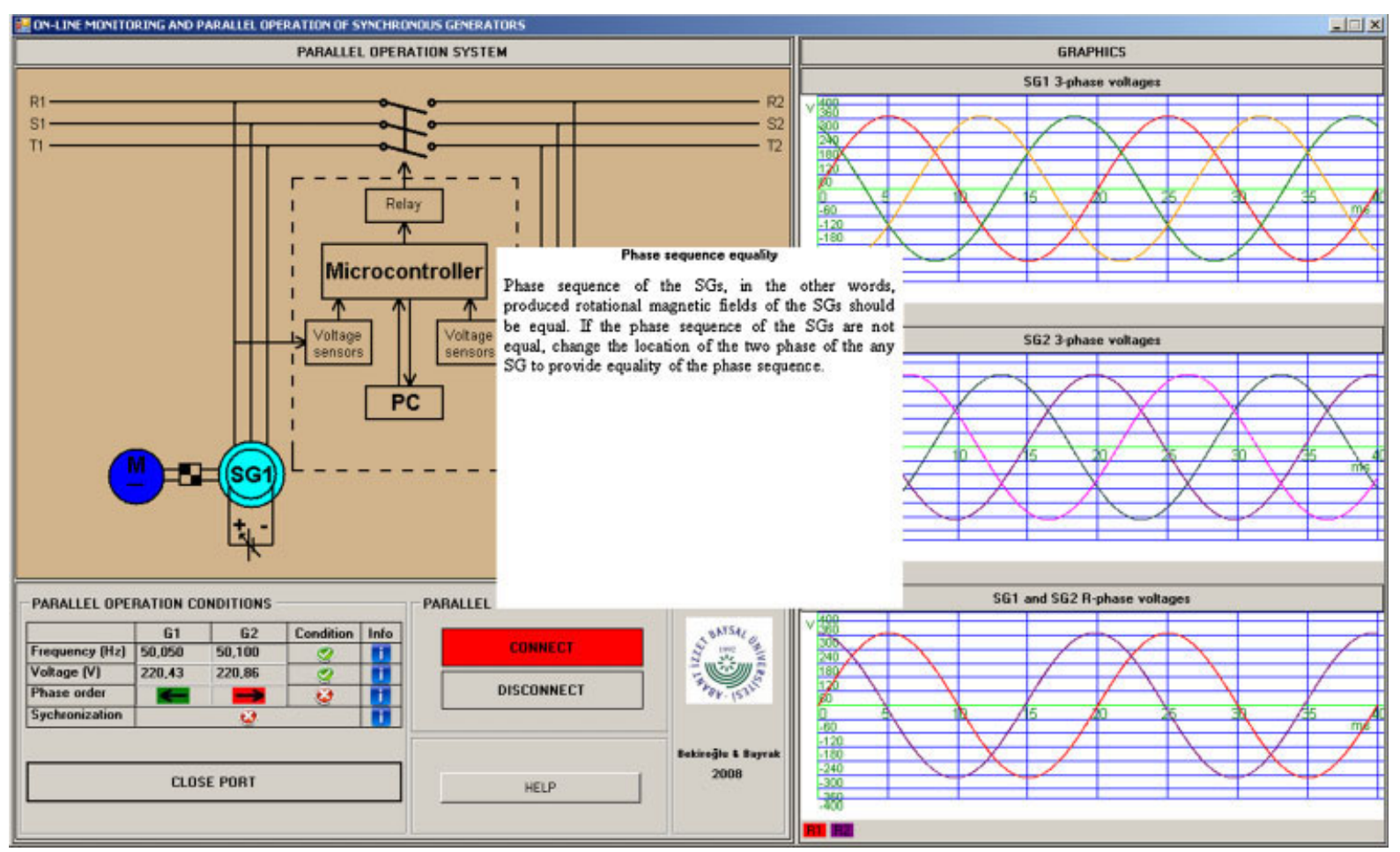

Figure 9 View of the educational tool for the Case 2. [Color figure can be viewed in the online issue, which is available at wileyonlinelibrary.com.]

\section{Case 3}

The system is operated and three of the parallel conditions are obtained and monitored on the educational tool as shown in Figure 10. The frequency, the voltage, and the phase sequence conditions are provided and the Correct signs are appeared for each condition on the educational tool. However, the synchronization is still not provided. And the Stop sign is appeared for this condition. The Connect button is activated and it is Green in this case. The system is ready to be connected in parallel. If the Connect button is clicked, the microcontroller will search for synchronization and will connect the SGs in parallel.

\section{Case 4}

Finally, all of the parallel connection conditions are provided and the SGs are connected in parallel automatically with the developed control tool. The view of the educational tool in this case is given in Figure 11. After clicking the Connect button, the system captures the synchronization time and sends the parallel connection command to the hardware system. Two SGs are paralleled and the parallel connection switch on the educational tool is also closed to represent the parallel operation of the SGs visually. In this case, the SGs are operated as single bus. The phase voltages and the frequencies of the SGs are equal. The phase voltages of the SGs are synchronous and there is no phase difference between the phase voltages of two SGs. This can be clearly seen on the graphics window.

If any parallel connection condition is removed for any reason, the educational tool is automatically switch-off the parallel connection of the SGs. The educational tool continues to measure and monitor the data of the SGs.

\section{CONCLUSIONS}

An on-line educational tool for monitoring and control of the parallel operation of the SGs has been designed and implemented. Hardware system consists of two SGs rotated with the DC motors has set-up to test the educational tool. Simple, precise, and compact control card has been designed for the measurements. There is no need for interface electronics circuits for processing, converting, and transmitting signals to the microcontroller. All signals are transmitted to the microcontroller via the signal transformers. The microcontroller calculates the phase voltages, the frequencies, and the phase sequences of the SGs according to received signals. Measured data are monitored on the educational tool on-line.

The measured values of the two SGs are compared to observe the parallel connection conditions. The actual status of the conditions are tested and viewed visually on the PC tool. Also, the voltage graphics of the SGs are plotted on the educational tool. The phase angle between voltages of the SGs can be followed with these graphics. The parallel operation of the SGs is controlled via the educational tool.

The developed educational tool is tested for the different operating cases. Obtained results showed that the developed tool is exact, fast, and reliable. It is independent from extra measurement devices. As a result, the developed tool can be used successfully for the parallel operation of the SGs with efficient cost. The educational tool is clear and user-friendly that can be 


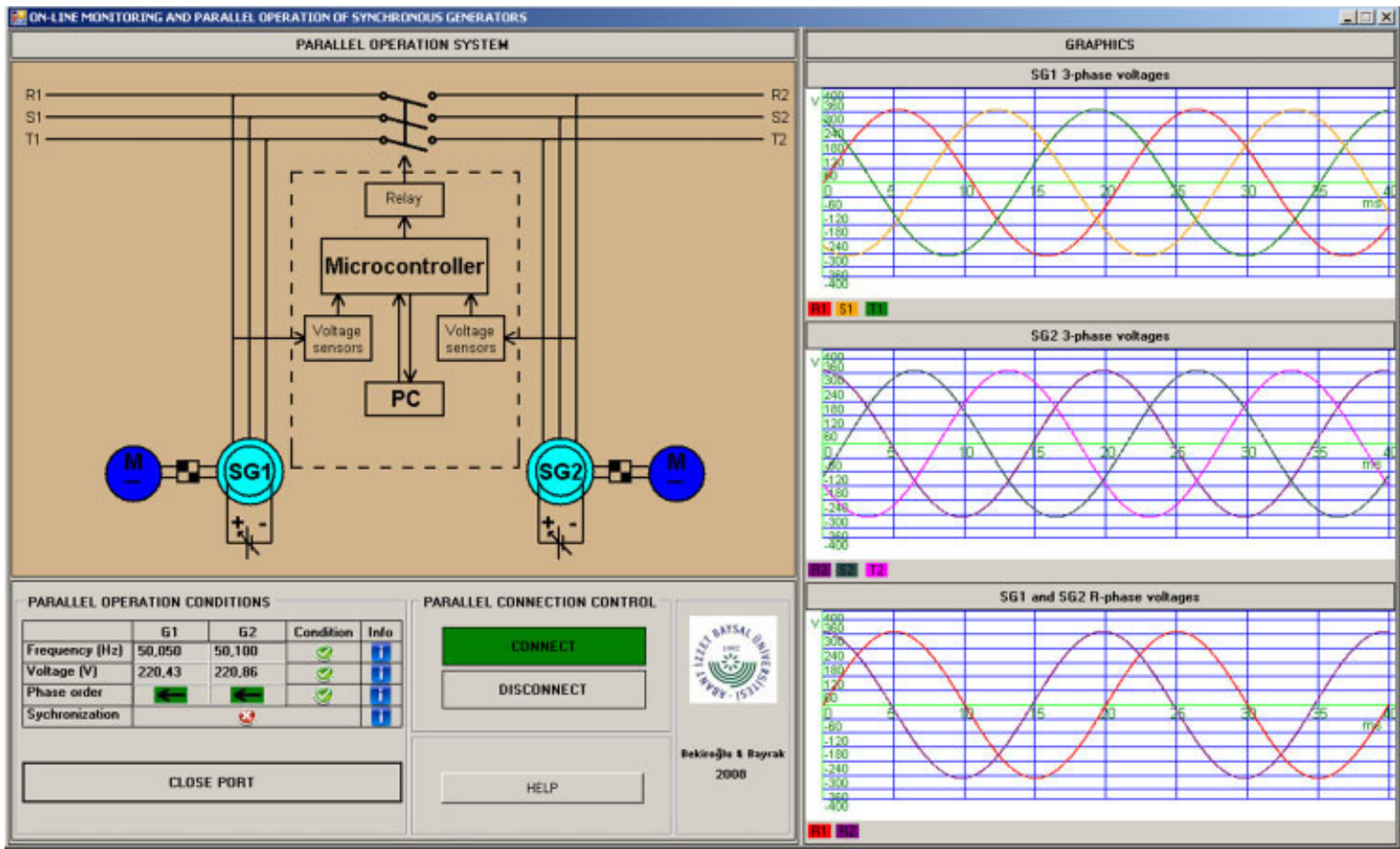

Figure 10 View of the educational tool for the Case 3. [Color figure can be viewed in the online issue, which is available at wileyonlinelibrary.com.]

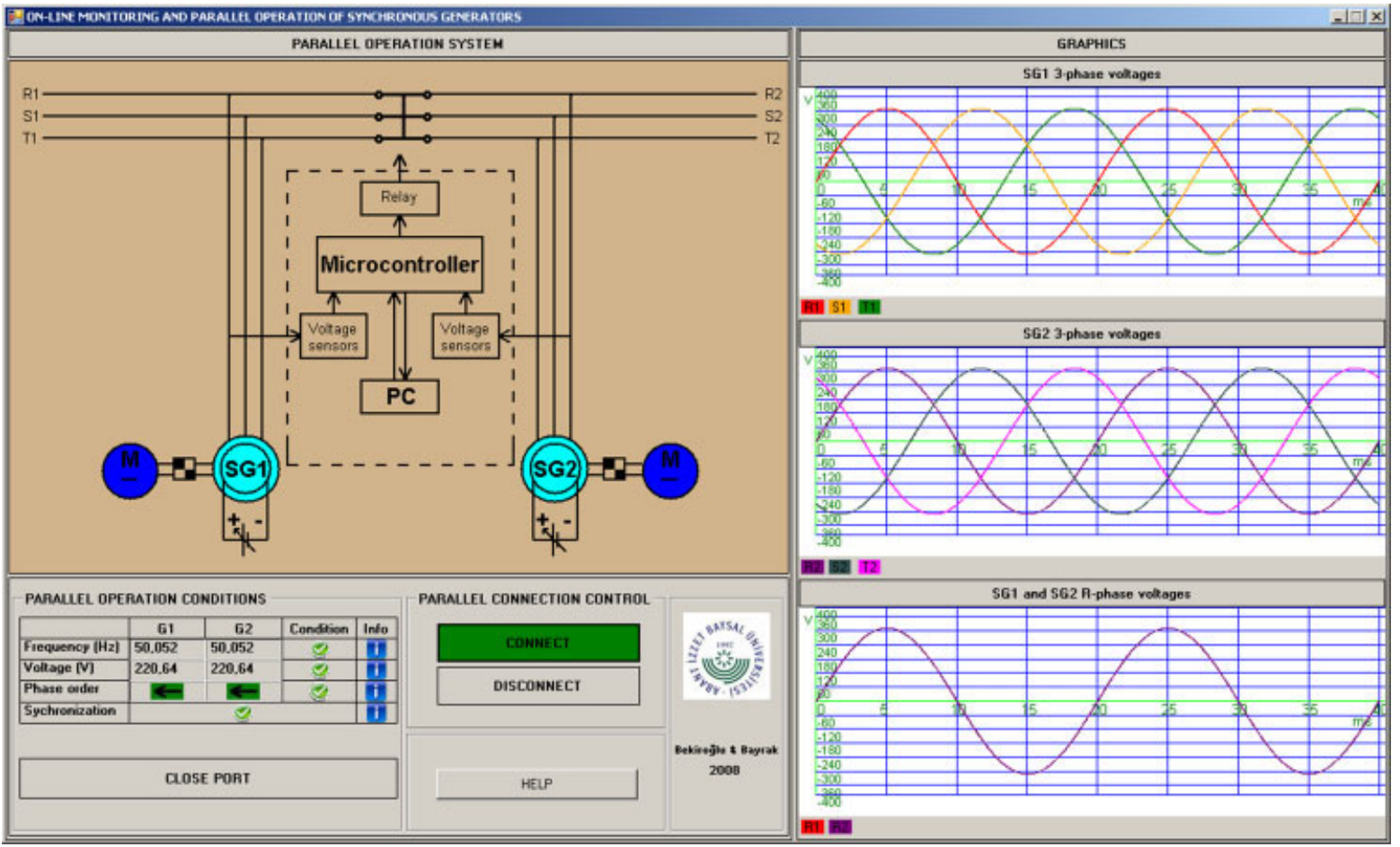

Figure 11 View of the educational tool for the parallel operation case (Case 4). [Color figure can be viewed in the online issue, which is available at www.interscience.wiley.com.] 
used easily and effectively for both industrial and educational purposes. Since students/engineers have facility to perform actual experiments, the proposed tool can prepare them for the design, build, test, and use real systems related to the SGs.

\section{ACKNOWLEDGMENTS}

This study was supported from the Scientific Research Project Commission of the Abant Izzet Baysal University, under grant 2006.01.09.252.

\section{REFERENCES}

[1] A. E. Fitzgerald, C. Kingsley, and S. D. Umans, Electric machinery. McGraw-Hill Book Company, Singapore, 1992.

[2] I. Colak, Synchronous machines. Seckin Press, Ankara, 2003 (in Turkish).

[3] M. S. Mahmoud and A. Ismail, Control of electric power systems, Syst Anal Model Sim 43 (2003), 1639-1673.

[4] H. M. B. Metwally, Operation of new variable speed constant voltage and frequency generator connected to the grid, Energy Conv Manag 41 (2000), 701-712.

[5] IEEE Std C37.118-2005, IEEE Power Engineering Society, IEEE standard for synchrophasors for power systems, 2005.

[6] I. Colak and E. N. Y̌ilmaz, Simulation of the parallel connection of electrical power stations, Int J Elect Eng Educ 36 (1999), 332-341.

[7] S. E. A. Emam, Automatic digital synchronization, International Conference on Electrical Electronic and Computer Engineering, ICEEC'04, 2004, pp 778-784.

[8] L. R. R. Montero, S. M. Wellington, and M. F. Gemerts, Monitoring and control system based on microcomputer for synchronous generator, IEEE Trans Energy Conv 14 (1999), 1424-1429.

[9] I. Sefa, M. Demirtas, I. Garip, and I. Colak, Design and implementation of parallel operation set of alternators for educational purpose, J Fac Eng Archit Gazi Univ 23 (2008), 729-739 (in Turkish).

[10] W. Kui, W. Siyuan, D. Gang, J. Li, Z. Xin, and P. Zhencun, Microprocessor-based multi-functional automation laboratory for power system, IEEE Conference on Industrial Electronics and Applications, ICIEA 2006, pp 1-5.

[11] T. W. Gedra, S. An, Q. H. Arsalan, and S. Ray, Unified power engineering laboratory for electromechanical energy conversion,

\section{BIOGRAPHIES}

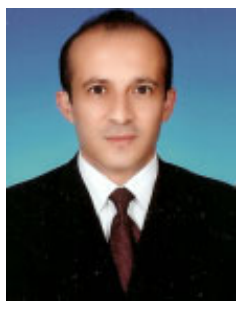

Erdal Bekiroglu received his BSc, MSc, and $\mathrm{PhD}$ degrees in the electrical technologies education from Gazi University. He worked as a research assistant at Gazi University between 1996 and 2003. He is currently assistant professor at the Department of Electrical and Electronics Engineering, Faculty of Engineering and Architecture, Abant Izzet Baysal University. His research interests are computer-controlled systems, digital signal processors, ultrasonic motors, drive and control of electrical machines. power electronics, and power systems, IEEE Trans Power Syst 19 (2004), 112-119.

[12] F. S. Sellschopp and M. A. Arjona, An automated system for frequency response analysis with application to an undergraduate laboratory of electrical machines, IEEE Trans Educ 47 (2004), 57-64.

[13] S. Li and R. Challoo, Restructuring an electric machinery course with an integrative approach and computer-assisted teaching methodology, IEEE Trans Educ 49 (2006), 16-28.

[14] A. Keyhani, M. N. Marwali, L. E. Higuera, G. Athalye, and G. Baumgartner, An integrated virtual learning system for the development of motor drive systems, IEEE Trans Power Syst 17 (2002), 1-6.

[15] A. Zamarron, M. A. Arjona, F. Briz, and J. M. Guerrero, A virtual instrument for detecting spatial saliencies: An electric machinery laboratory experience, Comput Appl Eng Educ 16 (2008), 62-71.

[16] S. Ayasun and G. Karbeyaz, DC motor speed control methods using Matlab/Simulink and their integration into undergraduate electric machinery courses, Comput Appl Eng Educ 15 (2007), 347-354.

[17] S. Ayasun and C. O. Nwankpa, Induction motor test using Matlab/ Simulink and their integration into undergraduate electric machinery courses, IEEE Trans Educ 48 (2005), 37-46.

[18] H. C. Lin, Web-based remote online maximum wind power monitoring and control system, Comput Appl Eng Educ 15 (2007), 155-165.

[19] M. Gokbulut, C. Bal, and B. Dandil, A Virtual electrical drive control laboratory: Neuro-fuzzy control of induction motors, Comput Appl Eng Educ 14 (2006), 211-221.

[20] O. M. Hernández, A. R. De La Rosa, D. Báez-López, R. Alejos, and E. Enríquez, Power Lab: A tool to learn electrical machines and power electronics, Comput Appl Eng Educ 7 (2000), 213220

[21] M. Gokbulut and A. Tekin, An educational tool for neural network control of brushless DC motors, Int J Eng Educ 22 (2006), 197-204.

[22] M. A. Akcayol and T. Yigit, A computer-based educational tool for pulse width modulator for static converters, Comput Appl Eng Educ 12 (2004), 215-223.

[23] S. P. Carullo, R. Bolkus, J. Hartle, J. Foy, C. O. Nwankpa, R. Fischl, and J. Gillerman, Interconnected power system laboratory: Fault analysis experiment, IEEE Trans on Power Syst 11 (1996), 1913-1919.

[24] C. K. Gan, A. A. Rahim, A. Maaspaliza, and M. Zharif, Virtual instrument-based synchronisation system, 4th Student Conference on Research and Development SCOReD 2006, pp 275-279.

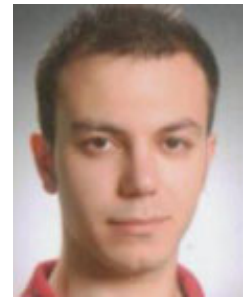

Alper Bayrak received his BSc and MSc degrees in Electrical and Electronics Engineering Department from Karadeniz Technical University and Gazi University, respectively. He worked as research assistant at Bolu Abant Izzet Baysal University, between 2005 and 2006 and at Gazi University between 2006 and 2007. He has been working at Izmir Institute of Technology since 2007. His research interests are computer-controlled systems, robotics, microprocessors, and biomedical image analysis. 